\title{
Inkjet Printing of Microcomponents: Theory, Design, Characteristics and Applications
}

\author{
Chin-Tai Chen \\ Department of Mechanical Engineering, \\ National Kaohsiung University of Applied Sciences, Kaohsiung \\ Taiwan, ROC
}

\section{Introduction}

Inkjet printing technology has been invented and used in the form of continuous jets for typewriting and recording over 50 years. However, this kind of printing technique becomes truly popular within twenty years for the public at homes and offices greatly thanks to the consumer products of desktop inkjet printers developed and marketed in the middle of the 1980s. Those leading manufacturing companies including Hewlett-Packard (HP), Canon, and Seiko Epson et al. have been celebrated around the world for the evolution of the modern digital printers since then. Compared to the mechanical typewriters in the past, the new inkjet printers perform the printing by dye or pigment-based ink droplets jetted through micro-electro-mechanical actuators, which feature non-impact and digital-control merits, thereby generating less noise and power consumption. Furthermore, as operating in called drop-on-demand (DOD) mode, their droplets with sub-nanoliter volume form the dot-matrix patterns onto medium surfaces, rendering static images for human sight with resolution from low to more than 1200 dot per inch (dpi).

To achieve high quality ink printing on various substrates, dispensing the micro droplets precisely from nozzles to underlying medium propose some technical difficulties concerning fluidic issues such as the variations in droplet volume, flight direction, and deposition morphologies. In terms of microfluidic flow, a stream of micro droplets significantly undergo high speed flying in air, impacting on a solid substrate, forming and drying above a substrate surface. Through the whole process, the issues about transition and stability of flow from liquid channels to individual droplets, and vice versa, will be frequently encountered in theory, which need to be delicately dealt with in advance of various applications. For instance, undesirable satellites from main droplets may be generated due to Rayleigh instability; similarly, it is possible to form irregular bulges within a lengthy liquid channel on a nonpenetrable substrate (e.g., glass substrate) instead of porous paper. Moreover, nonuniform evaporation can be caused in nature for volatile sessile droplets placed on flat surfaces, in which the called coffee-ring effect often seen for diluted fluids (Deegan et al., 1998) should be avoided for the requirements of uniform thickness. Besides the conventional dye and pigment inks, almost the materials in solution involve the complexity of evaporable droplets, when applying to the inkjet printing processes that rely heavily on the full understanding of droplet behaviors on various surfaces from wet to dry stages. 
Nevertheless, in the recent years, much attention of studies in academics and industries as well is paid to the uses of various materials in the inkjet printing processes, including nanoparticle metals (Szczech et al., 2002; Kang et al., 2010), bio-chemicals (Busato et al., 2007; Fuchs et al., 2011), colloidal polymers (MacFarlane et al., 1994; Biehl et al., 1998; Jeon et al., 2005; Chen et al., 2008), coloring and light emitting materials (Bale et al., 2006; Chen et al., 2010; Chang et al., 2011), transistors and semiconductors (Sirringhaus, et al, 2000; Han et al., 2009; Hinemawari et al., 2011), and so forth. Compared to conventional two deposition techniques, i.e. physical vapor deposition (PVD) and chemical vapor deposition (CVD), this current inkjet printing has been considered as alternative third one that can be termed here droplet vaporization deposition (DVD). The DVD method (Chen et al., 2011) further provides capability of direct patterning on substrates without the employment of photolithography, and therefore becomes a very promising green technology that will consume fewer amounts of material and power with minor waste compared to both the formers as mentioned earlier. Therefore, using the inkjet printing as a digital microfabrication tool, many associated manufactures in the world have developed the inkjet printing platforms and equipments from the laboratory to commercial grade, for example MicroFab Inc. (www.microfab.com) and Dimatix Inc. (www.dimatix.com), in order to provide the required technology services for research and pilot production.

With the above inkjet printing materials and equipments required, many different processes and strategies have consequently been followed to directly print specific products by applying this tooling method. For example, the manufacturing apparatus of inkjet-printed color filter that has been one of key components for liquid crystal display (LCD) was first proposed and patented by Canon Company (Satoi, 2001). Primary three colors of red (R), green $(\mathrm{G})$, and blue (B) can be firmly formed on the receptor layer of a substrate through a series of RGB coloring, ink fixing, and curing of receptor layer, where the substrate requires film coating for absorbing the color inks prior to inkjet printing. Based on the this kind of application, more persisting efforts were made within recent years about the improvement of ink formulas (Kim et al., 2009; Chang et al., 2011), surface treatments and characteristics (Koo et al., 2005; Chen et al., 2010), and large-area and active lighting (Bale et al., 2006). In a similar development way, more extensive studies for inkjet printing applications were also directed to other fields including biochips (Xu et al., 2005; Gutmann et al., 2005), optical microlenses (Nallani et al., 2006; Chen et al., 2009), microelectromechanical systems (Fuller et al., 2002; Cho et al., 2006; Alfeeli et al., 2008), and electronic components (Lee et al., 2005; Scandurra et al., 2010; Perelaer et al., 2010). Among them, many of the fundamental processes (e.g., surface treatments and droplet depositions) and components (e.g., inkjetprinted polymers and conductors) are in common with the present display applications, in particular the polymer light-emitting and liquid crystal displays (Katayama, 1999; Mentley, 2002; van der Vaart et al., 2005). Therefore, full understanding of inkjet printing microcomponents is a substantial route to produce the key components for the applications of display based on the inkjet printing techniques.

This chapter will comprehensively report the microfabrication techniques and applications for various microcomponents via inkjet-printing processes over decades, especially used for the present and future liquid crystal displays. It is organized briefly as follows. The background of current research and development about the inkjet printing technique is introduced here in Section 1, followed by the technical strategy of inkjet printing processes detailed with the concentrated respects of critical requirements for materials and facilities in Section 2. Section 3 addresses the critical design issues of microfabrication such as the 
position accuracy and morphology formations required to be satisfactory for realizing mass product. Section 4 describes the discovered characterization of droplet depositions involved mostly with liquid evaporation, solute deposit, and patterns in geometries. Section 5 presents the concept-proof applications of the inkjet-printing processes, elaborating their present technical progresses and challenges in the future, which are intimately related to the display components including color filters, polymer light emitting diodes, microlenses and backlight planes, conductive lines and electrodes, transistors and integrated circuits. Finally, we draw a conclusion in Section 6.

\section{Strategy of inkjet printing processes}

To fulfill the specific requirements of applications, basic inkjet printing processes should be comprehensively designed and realized with four major elements of system implementation: ink materials, substrate properties, droplet generation, electromechanical platforms and printing algorithm. As shown in Figure 1, each element involves different technical considerations and plays a critical role of a whole inkjet printing system for realizing the process. Those key elements of fulfilling the inkjet printing components will be thoroughly described and discussed in the next paragraphs.

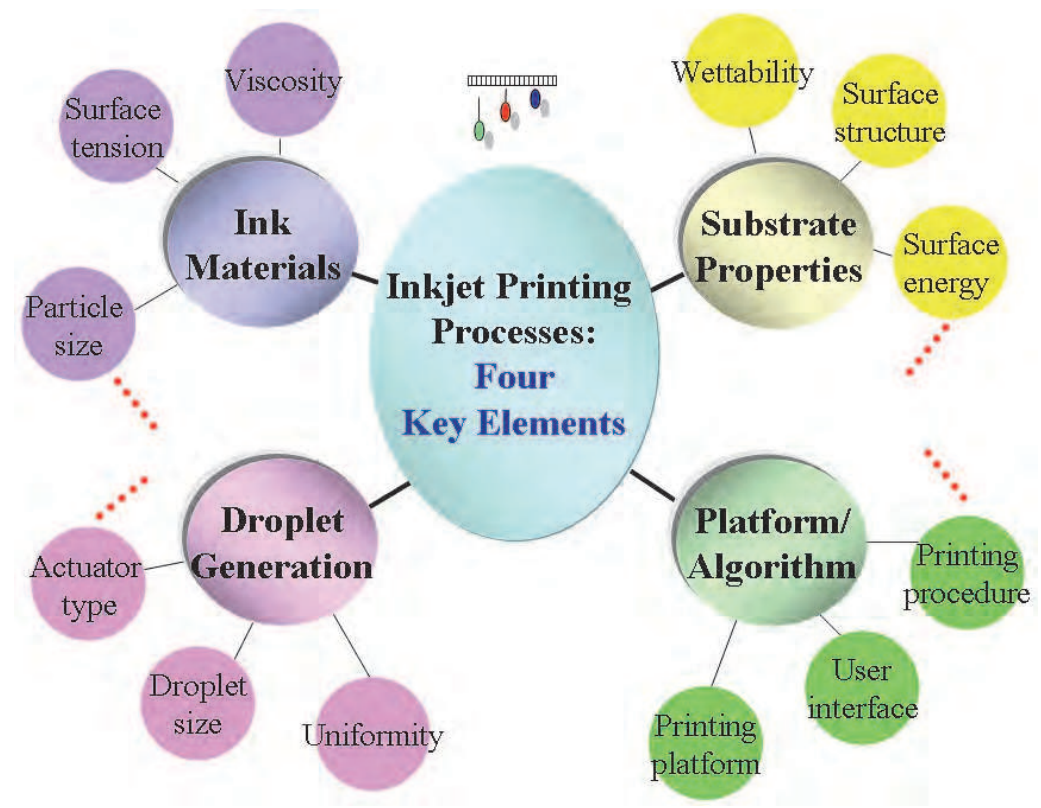

Fig. 1. Major elements of system implementation in strategy of inkjet printing processes including ink materials, substrate properties, droplet generation, and electromechanical platform combined with printing algorithm.

\subsection{Ink materials}

For any application, appropriate selection of inks is firstly important as the primary consumable during inkjet printing, which is a liquid solution generally composed of solvent 
and solute. To be stored stability within a printhead reservoir, the solid contents (i.e., solute $\sim 5-25 \mathrm{wt} \%$ ) of ink materials have to be uniformly dispersed in the solvent by additional suitable surfactant, in which no sedimentation is required for the long run duration (de Gans et al., 2004). And the ink viscosity needs to be low sufficiently for jettability of droplet actuators as well. Hence, the surface energy $(\gamma)$ together with dynamic viscosity $(\mu)$ of ink solutions depending on various printheads are typically in the range of $25-55 \mathrm{mN} / \mathrm{m}$ and 3$10 \mathrm{mNs} / \mathrm{m}^{2}(\mathrm{cp})$, respectively, where pure water has $\gamma$ of $\sim 72 \mathrm{mN} / \mathrm{m}$ and $\mu$ of $1 \mathrm{cp}$ at $20^{\circ} \mathrm{C}$. The sub-micrometer size of suspended particles, and in particular pigment and metal particles, is also limited to extent that is determined by some thresholds (e.g., filter cavity and nozzle neck) inside the micro liquid channels of printheads.

Besides, heating issues of ink materials should be considered when thermal bubble printheads are used instead of dominate piezoelectric ones. For instance, some biological materials (e.g., DNA and proteins) and polymers (e.g., UV-curable photoresists) may be sensitive to the elevated temperature (up to over $200^{\circ} \mathrm{C}$ ) during tens of microseconds of heating pulses, although a few studies on epinephrine, collagens (Chiu et al., 2006) and UV color-resists (Chang et al., 2004) were demonstrated successfully by bubble inkjet printing. More different types of ink materials, for example, the conductive nanoparticle metals or polymers in bubble jetting (Shaw \& Seidler, 2001), are still unclear for the heating sustainability when curing and sintering at higher temperature as required. Since thermal printheads are much less expensive than piezoelectric ones (Tseng et al., 2002), it deserves to be further investigated for versatile materials, in view of the low-cost disposable circumstances.

\subsection{Droplet generation}

Filled with inks of suitable properties, a stream of droplets can be expelled from tiny nozzles of reservoirs on demand when the printhead is driven by electric pulses. Based on different DOD types of printheads (Le, 1998), such as thermal, piezoelectric, electrostatic, and acoustic ones, those droplets generally undergo morphological transition due to interplay of viscous force and surface tension, from initial to necking stages as demonstrated in Figure 2. Thus the volume of formed droplet is estimated as

$$
V=\pi d^{3} / 12+\pi d^{2} h / 12
$$

where $d$ is the diameter and $h$ is the height for the drop, respectively. In principle, the larger surface energy liquid possesses during ejection, the smaller drop $(V)$ is formed by shorter height $(h)$, thus producing finer width of a line on the substrate. In the practical case of $h=6 d$, for example, the final spherical diameter of droplet becomes 1.5 times the original size of main droplet, which is determined largely by the nozzle diameter. In general, the size of nozzle in diameter can range from $10 \sim 100 \mu \mathrm{m}$, thereby corresponding to the droplet volume with one to hundreds of picoliters ( $\mathrm{pl}=10^{-12}$ liter). Obviously, higher resolution of the inkjet printing is therefore yielded by reducing the printhead nozzle with smaller diameter.

In fact, except for printing resolution, the uniformity of individual droplets in directionality and sizing is another considerable issue for precise manufacturing of microcomponents (Chen et al., 2001). Positioning accuracy together with morphological formation of droplet deposition that will be discussed in next Section 3 can be significantly influenced by the deviation from the targeted values. And this technical difficulty, in some situations permitted (Chen et al., 2010), may be compensated in terms of tuning substrate properties and printing algorithms as follows. 

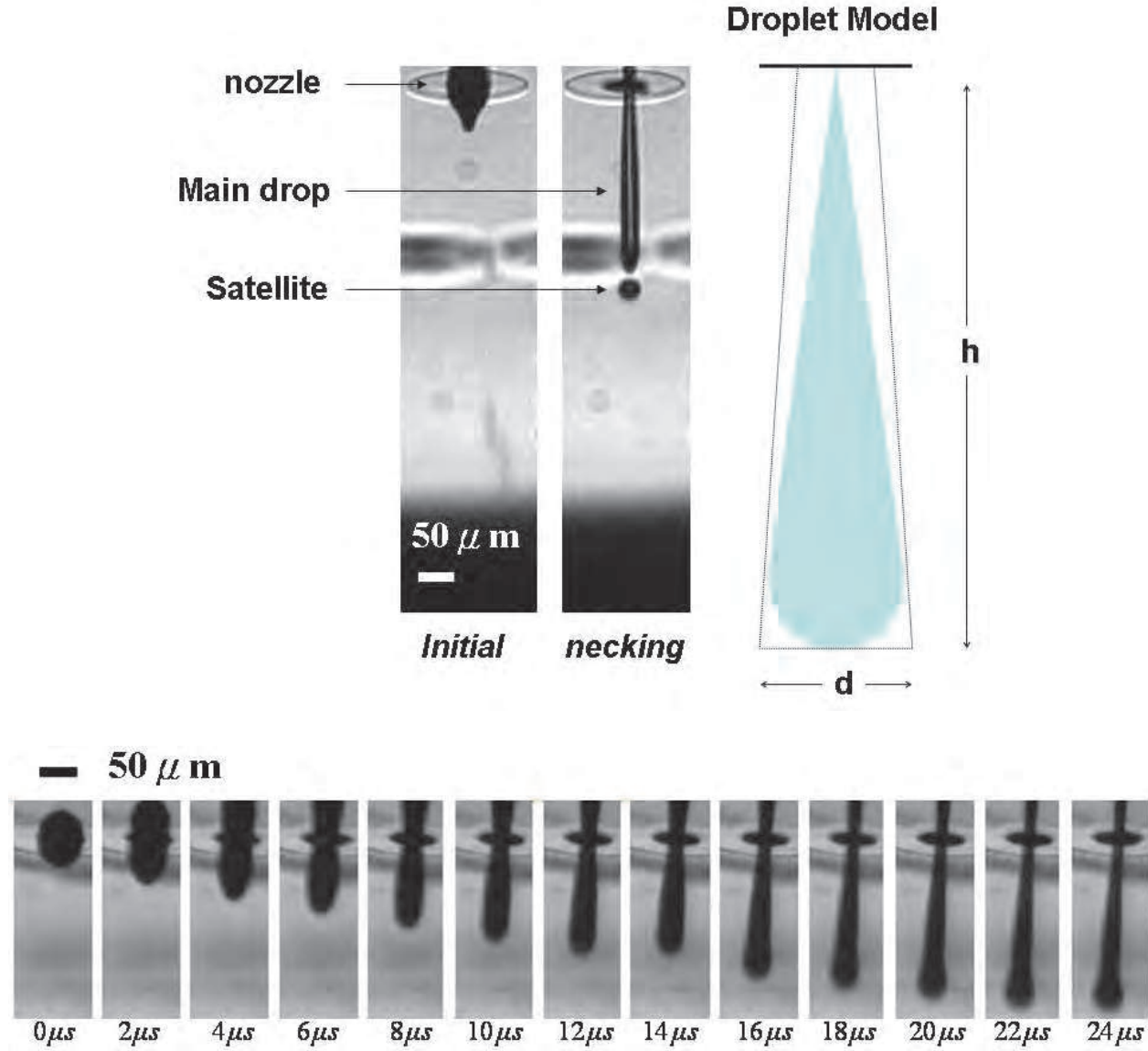

Fig. 2. (Top) Typical jetting process of a micro droplet generated through a tiny nozzle, from initial to necking stages; (Bottom) dynamic evolution of droplet formation in tens of microseconds.

\subsection{Substrate properties}

The conventional inkjet printers use regular paper made of fibers with porous top surface to absorb ink droplets. However, as illustrated in Figure 3(a), a glass surface can generate different deposition patterns from that on paper for the same ink, since free surface fluid flow is induced on such a solid surface. For a homogeneous surface, a deposited sessile droplet obeys a classic Young-Laplace (Y-L) relation as below (Pujado et al., 1972; Chen et al., 2008):

$$
\gamma_{s g}-\gamma_{s l}=\cos \theta_{c} \times \gamma_{g l}=\cos \theta_{c} \times \frac{\Delta P}{\left(R_{1}^{-1}+R_{2}^{-1}\right)}
$$


where $\gamma_{s g}, \gamma_{s l}$, and $\gamma_{g l}$ are the surface tensions of solid-gas $(s-g)$, solid-liquid (s-l), and gasliquid $(g-l)$ interfaces, respectively; $\theta_{c}$ is the contact angle of the $s-l$ interface, $\Delta P$ is the pressure difference across the $g-l$ interface, and $R_{1}, R_{2}$ are the two principal radii of the droplet curvature.

However, a different story can be given by many research groups for droplets on heterogeneous surfaces (Kim et al., 1995; Gau et al., 1999; Anton et al., 2000; Lenz et al., 2001; Bao et al., 2002), where the use of minimal free energy for self-assembly to form stable shapes was investigated in theory and experiments. For example, as demonstrated in Figure $3(b)$, pure water droplets spontaneously formed (self-assembled) the circular and stripe shapes on hydrophilic (glass) domains only of the structured surfaces, on which the glass were pre-patterned with hydrophobic (Teflon) domain by photolithography. Based on this concept of self-assembly on structured surfaces, stable solid formations of droplet deposition for striped channel (Chen et al., 2007) and circular shapes (Chen et al., 2009) were experimentally realized by inkjet printing of colloidal inks.
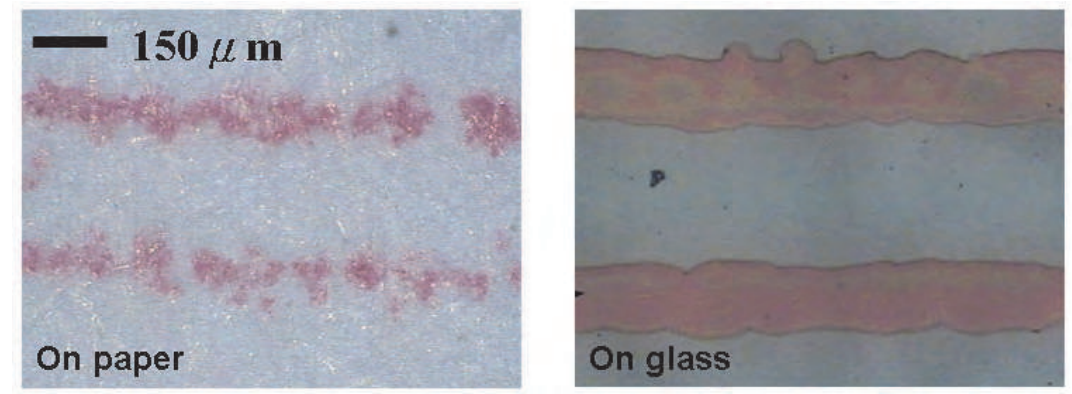

(a)
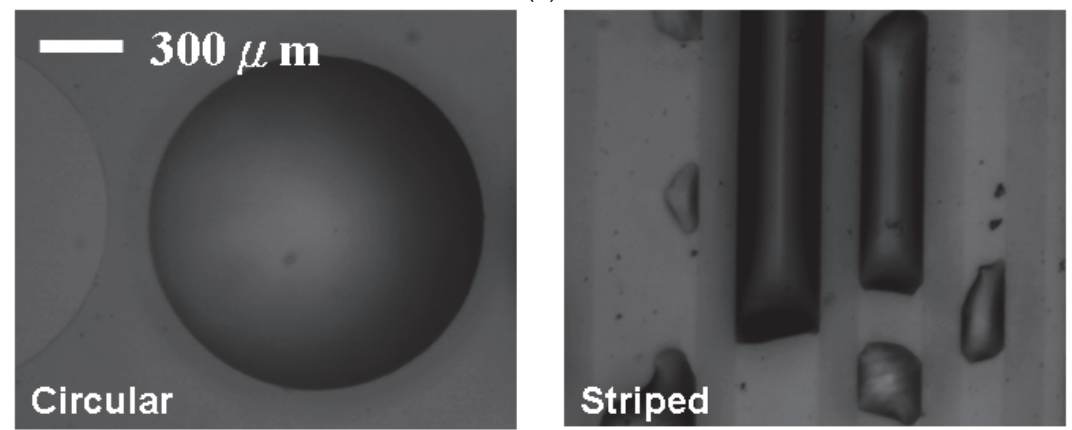

(b)

Fig. 3. (a) Dried formations of color inkjet printing on (porous: left) paper and (solid: right) glass, respectively; (b) wetting topography of pure water droplet formation on circular (left) and striped (right) Teflon-patterned glass surfaces.

\subsection{Inkjet printing platform and printing algorithms}

In recent years, there have been some microfabrication platforms particularly developed for the inkjet printing processes as mentioned above. As can seen in Figure 4, an inkjet printing platform prototype, laboratory-designed and developed for inkjet printing of color 


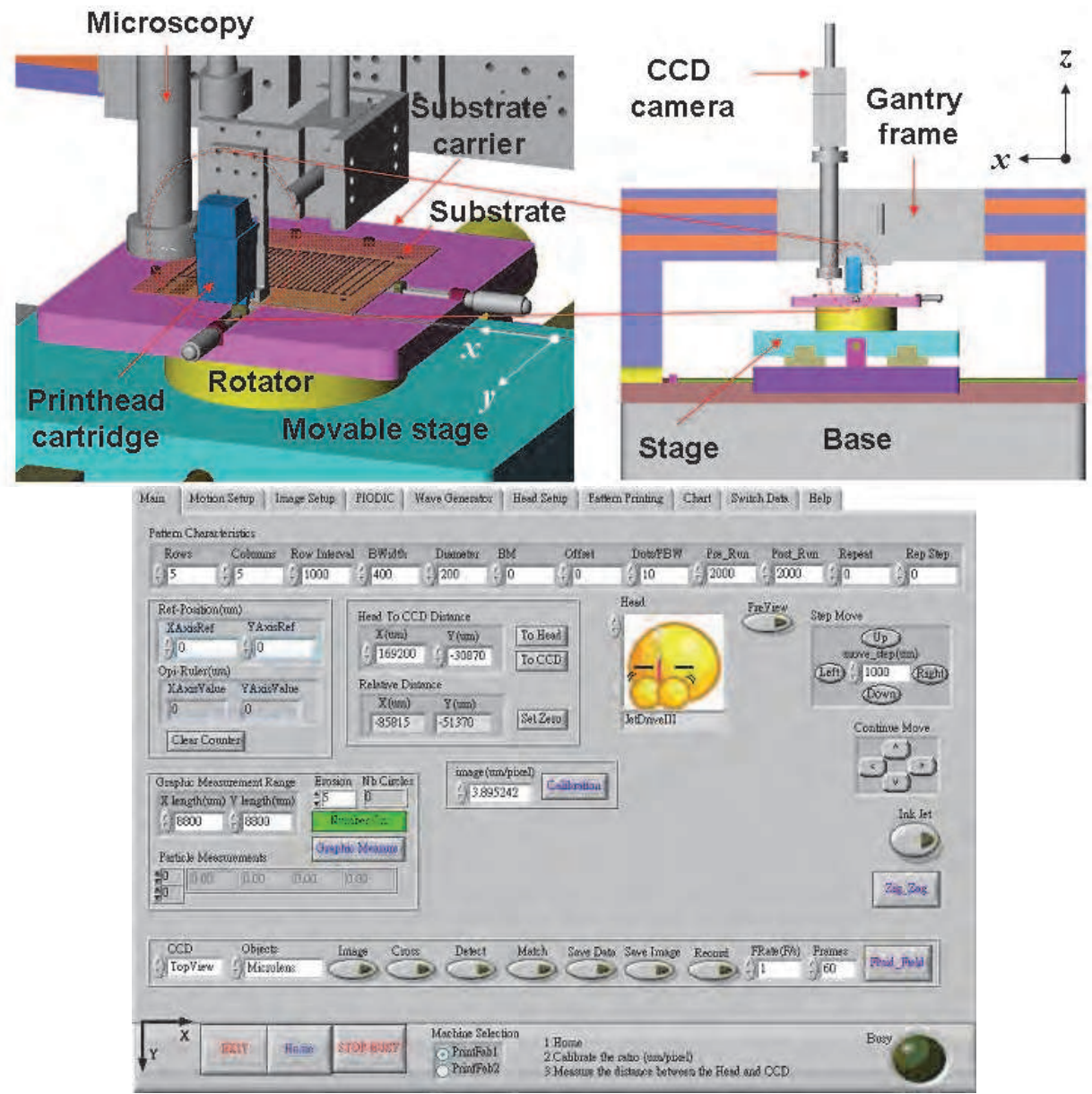

Fig. 4. (Right) Illustration of inkjet printing platform mainly composed of the printhead cartridge, movable stage, CCD camera, base, gantry frame, etc.; (Left) enlarged portion of the platform for highlight of the inkjet printing area; (Bottom) User interface window built by LabView in the controlling computer.

filters (Chen et al., 2010) by Industrial Technology Research Institute (www.itri.org.tw) in Taiwan, illustrates a common configuration primarily comprising the printhead cartridge, substrate carrier, movable stage and rotator, CCD camera and microscopy, base and gantry frame. With the printhead cartridge fixed on the gantry frame, this platform can perform DOD inkjet printing algorithm by moving the motored stage to deliver the substrate in $x-y$ coordinates so that the imaging pattern is online input and completed through a friendly user interface (PC-based LabView).

To achieve high quality inkjet printing, however, additional pre-printing procedures should be carried out in advance of actual inking the underlying substrates, which includes the $z$ axis gap tuning between the nozzle plate to substrate surface, cleaning of printhead nozzles, alignment and calibration of homing coordinates in $x-y$ axes (Huang et al., 2009), whereas 
special care is also paid for rotational registration if a large-area substrate (e.g., LCD color filter) is to be printed here.

After the full preparation of inkjet printing processes, two major design issues of microfabrication associated with precise DVD implementation will be further addressed and explained below.

\section{Design issues of microfabrication}

\subsection{Positioning accuracy}

Because of the jet instability in microfluidic nature, as demonstrated in Figures 2 and 3, most of droplets jetted from the nozzles exhibit slightly uncertain deviation of angle, $\Delta \theta\left(e . g ., \sim 1^{\circ}\right)$ from the normal direction of the horizontal plate. This uncertainty poses the issue of positioning precision for droplet deposition, which results in the inaccuracy of the location and width for the thin films formed on the substrates, as depicted in Figure 5. The rectilinear displacement on the surface nearly equals to $H \Delta \theta$, where $H$ (typically, $\sim 0.5-1 \mathrm{~mm}$ ) is the distance between the nozzle and the substrate surface (for example, it can amount to about $17 \mu \mathrm{m}$ comparable to the desired film width, i.e., $100 \mu \mathrm{m})$.

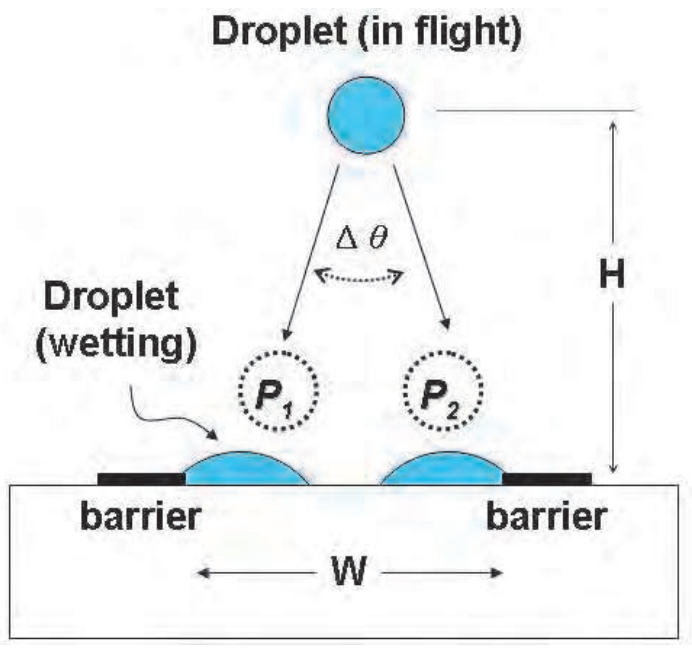

\section{Droplet wetting in a circular well (simulation)}

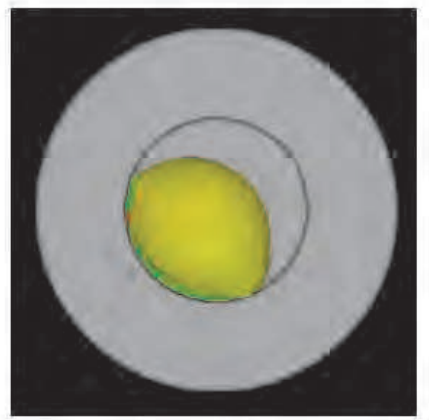

Fig. 5. (Left) Uncertain deviation angle of individual jetted droplet from the normal direction of the horizontal plate; (Right) microfluidic simulation of droplet wetting in a circular well.

Concerning the uneven width as well as positioning uncertainty, those difficulties can be surmounted using heterogeneous (structured) surfaces, as aforementioned previously. They have demonstrated the remarkable effect of registration on wetting and dried positions for droplets on heterogeneous glass substrates (e.g., Teflon coated and patterned on the surfaces). Those wetting droplets substantially exhibit minimum surface tension in the hydrophobic domain by repelling the other ones, leading to self-align along the surrounding rim (Joshi \& Sun, 2010). In fact, the wetting rim acts as a "virtual barrier" for droplets to resist flowing across the hydrophobic regimes. However, this energy-patterning strategy using thin-film coating technique suffers from the instability of liquid morphology imposing 
the limit of liquid volume to the droplets and thus making the thickness of the dried deposition film insufficient and nonuniform. Hence, another approach applying a concept of 'physical barrier' (Chen et al., 2010) was proposed to deal with the above constraints without losing the any positioning accuracy. In this case, as demonstrated in Figure 5, the simulated droplet was capable of self-aligning along the surrounding sidewall to prevent flowing over a circular well. Therefore, for a number of droplets, their allowable collective deviation of $H \Delta \theta$ can be raised to be $W$ at maximum (Chen, 2004).

\subsection{Morphology formation}

Another concern with the inkjet printed microcomponents involves the control of the morphology of the droplet deposition that is typically complex and varying in different situations. A deposited liquid droplet with volume of $V_{1}$ obeying the Y-L relation will simply form a hemispherical shape on homogeneous surface, with characteristic base radius $R_{b}$ of footprint as

$$
R_{b}=\frac{3 \cot \left(\theta_{c} / 2\right)}{2\left[3+\tan ^{2}\left(\theta_{c} / 2\right)\right]} \times V_{1}^{1 / 3}
$$

where $\theta_{c}$ is the contact angle as defined previously. Namely, the droplet footprint radius $R_{b}$ is proportional to its volume $V_{1}$ with scaling exponent of $1 / 3$; additionally, the smaller the contact angle $\theta_{c}$ is, the larger the footprint radius $R_{b}$ will be.

Furthermore, linear morphology from a number of droplets show more complicated than that of single dot, including individual-drop, scalloped, uniform, bulging, and stacked-coins formations, which are controlled by the delay and drop spacing as well (Soltman \& Subramanian, 2008). As the evaporation and curing temperature involved (Biswas et al., 2010; Scandurra et al., 2010), their morphology formations will change dramatically with more complexity in geometry and structure that will be further discussed in next Section 4 .

\section{Characterization of droplet deposition}

Two key dimensionless parameters describe the hydrodynamics of droplet deposition: the Reynolds number $(R e)$ and Weber number $(W e)$. Typically, supposed the values of $U$ ranging from one to ten meters per second (i.e., 1-10 m/s), the Reynolds number (Re, a ratio of inertia force to viscous force) gives corresponding values of 2 to 277 , which is small sufficiently to render the laminar flow (typical requirement of less than 2300). Also, the Weber number (We, a ratio of inertia force to surface-tension force) yields the corresponding values of 0.36 to 320 ensuring the final formation of droplet (Liou et al., 2008). Moreover, the droplet on substrate surface dynamically evolves into three distinct stages in succession: impacting, spreading (and wetting), and drying, as shown in Figure 6. As a result, the droplet deposition of interest for practical applications can be further discussed and characterized in three respects in the following.

\subsection{Evaporation deposit}

Over the last decades, evaporation kinematics of a pure droplet, without solid content involved, on homogeneous surface were thoroughly investigated, in theoretical and experimental ways, for various conditions (e.g., droplet and substrate materials), in which all mostly featured highly nonlinear (hysteresis) behaviors for the rates of contact angle, base 


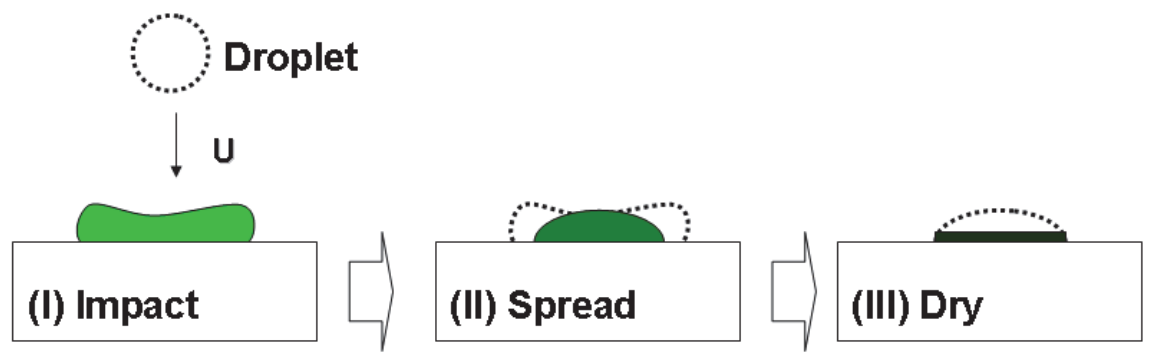

Fig. 6. Hydrodynamic evolution of a droplet on substrate surface through three distinct stages in succession: (I) impacting (II) spreading, and (III) drying.

radius and height (Bourges-Monnier \& Shanaha, 1995; Decker \& Garoff, 1997; Erbil et al., 2002; Hu \& Larson, 2002; Chen et al., 2006). A the same time, solution droplets that contain either suspended particles or colloidal polymers exhibit more complex fluidic properties (induced flows) due to such non-uniform evaporation (Adachi et al., 1995; Parisse \& Allain, 1997; Conway, et al., 1997; Gorand et al., 2004). One significant breakthrough in theories and experiments for evaporation deposit was disclosed by Deegan et al. (Deegan et al., 1997), with a derived expression of evaporative flux $J(r)$ under a small contact angle as

$$
J(r) \propto \frac{1}{(R-r)^{1 / 2}}
$$

where $R$ is the droplet base radius with contact line fixed on surface, and $r$ is the radial distance from the center of the droplet. Radial liquid flow towards the droplet side is induced during evaporation, thereby carrying the suspended particles within the droplet to its surrounding that was termed coffee-ring (CR) effect. As can be seen in Figure 7, an aqueous PVA (Polyvinyl Alcohol) 20\% droplet formed non-uniform surface profile after drying, due to this remarkable $\mathrm{CR}$ effect, showing a characteristic concave shape that the perimeter region was much thicker than the center one over three times (i.e., $12 \mu \mathrm{m} / 4 \mu \mathrm{m}=3$ ).

Some research efforts to avoid the non-uniform droplet deposit were recently reported (Chang et al., 2004; Chen et al., 2004; Weon \& Je, 2010), since the deposit thickness is important for many applications such as biochips, LCD color filters, and light-emitting displays. Among them, special treatment on either homogeneous or heterogeneous surfaces plays a critical role on controlling the final deposit formations during evaporation, because of pinning or de-pinning condition as boundary constraints (Chen et al., 2009).

\subsection{Deposit patterns and properties}

As a whole, deposit patterns that fulfill the duplication from virtual (digital) codes in computers to real (printed) formations on substrates can be rendered and featured in geometry, including two-dimensional (planar) dot matrix, one-dimensional (linear) stripes, and arbitrary images. Those digital patterns can be dealt with in various formats: either text (e.g., location coordinates) or drawing ones (e.g., bmp, jpg). For example, as shown in Figure $8(a)$, a typical dot-matrix $(150 \times 200)$ covering a rectangular region can be formed by PU (Polyurethane) 15\% droplets on the hydrophobic (Teflon-coated) substrate, in which each individual $173 \mu \mathrm{m}$-diameter dot with spacing of $450 \mu \mathrm{m}$ was inkjet-printed to exhibit uniformly hemispherical. Rather, on hydrophilic glass surface, as demonstrated in Figure 

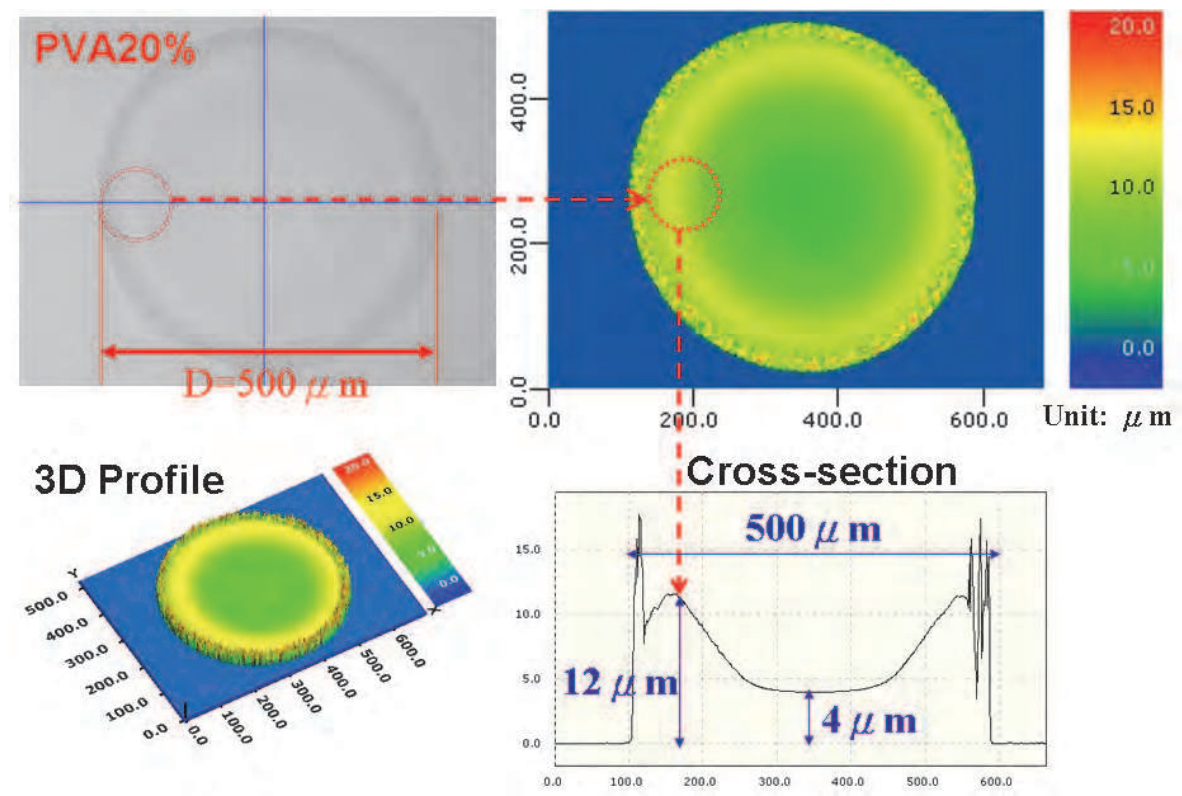

Fig. 7. Evaporation deposit of an aqueous PVA $20 \%$ droplet on homogeneous glass characterized with apparent coffee-ring effect on nonuniform surface profile.

8(b), simple straight lines were self-formed by Ag (silver) nanoparticle inks when the smaller dot spacing of $5 \mu \mathrm{m}$ was used. As further proceeding, any arbitrary images, like cartoon Doraemon as depicted in Figure 8(c), were carried out with ease demonstrating versatile capabilities of image processing in inkjet printing.

As a matter of fact, this allowable versatility of deposit patterns exactly offer such a unique advantage of material and time saving as a cost-efficient technique compared to the conventional others. Hence, different evaporation depositions and patterns can be selected for specific applications. Also, their corresponding properties such as optical, mechanical, and electronic performances depend solely on the technical requirements of specifications in commercial products. For instance, the dot-matrix as shown in Figure 8(a) can be used a microlens array such that optical transparency is dominant, whileas the electric conductivity should be emphasized for the straight lines in Figure $8(\mathrm{~b})$ being used as the conductors in circuitry.

Therefore, typical inkjet printing applications, insofar as potentially useful candidates for electric display fields, will be described and explained in the next paragraph.

\section{Applications}

\subsection{Color filters}

Generally, LCD color filters (CFs) feature a dot-matrix with primary red (R), green (G), blue (B) colors. Each color dot presents a tiny pixel of the full-color display with characteristic size ranging from tens to hundreds of micrometers, which match the droplet size if a highresolution inkjet printing process is applied. Thus, much research has been done in the development of the inkjet-printed color filters, including the suitable UV-curable inks and 

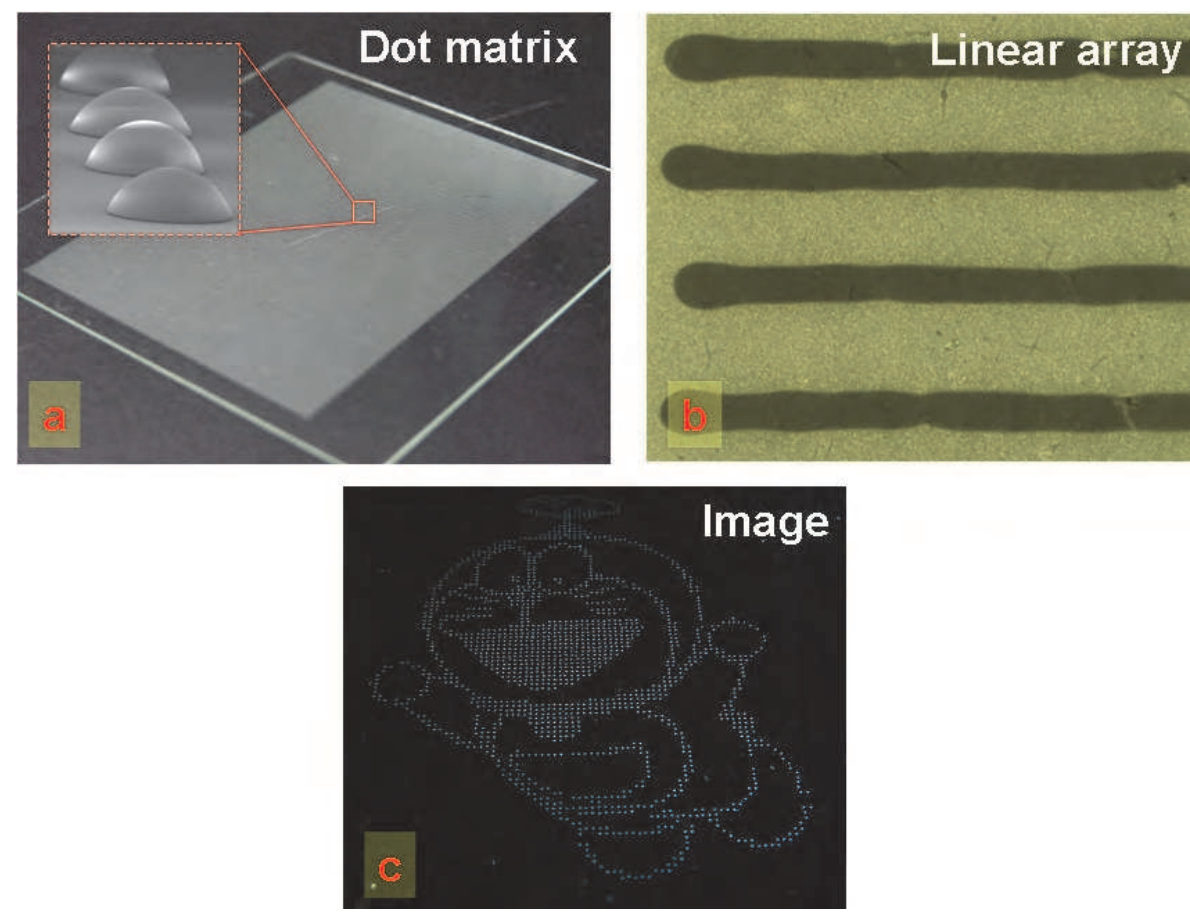

Fig. 8. (a) Individual convex $173 \mu \mathrm{m}$-diameter PU deposits inkjet-printed in a $150 \times 200$ matrix on a $10 \times 10 \mathrm{~cm}^{2}$ Teflon-coated glass, (b) linear Ag-nanoparticle deposits of $\sim 200 \mu \mathrm{m}-$ width inkjet-printed on glass surface, (c) inkjet-printed cartoon Doraemon on glass surface.

novel printing platforms (Satoi, 2001; Chang et al., 2005; Koo et al., 2006; Chen et al., 2010), to replace the conventional techniques based on photolithography. Figure 9 shows an inkjetprinted stripe-type color filter with RGB thin-film layers built on the underlying blackmatrix (BM) glass, where the sidewalls were pre-patterned by photolithography to prevent the overflows between different color inks (Chen et al., 2010).

Although great success of inkjet-printed color filters was achieved in some respects, there are challenging issues, including higher color density, reliability and yield rate, to be further resolved in future mass production. At the same time, the similar inkjet printing processes have been adopted for active-lighting components, polymer light-emitting-diode (LED) displays, which are described as below.

\subsection{Polymer light emitting diodes}

Instead of performing light-filter in CFs, polymer light emitting diodes (LEDs) serve as the active-matrix components for lighting without back light required for CFs. As conjugated polymer materials used for electroluminescence that are commercially available (www.cdtltd.co.uk), the polymer LEDs can be directly applied for full-color displays using the inkjet printing technique (van der Vaart et al., 2005; Bale et al., 2006). Since the LED materials are sensitive and degenerative via chemical reactions (e.g., for water $\mathrm{H}_{2} \mathrm{O}$ and oxygen $\mathrm{O}_{2}$ ), their productions through inkjet printing processes require delicate control of background environment when the droplet depositions of conjugated polymer materials are 

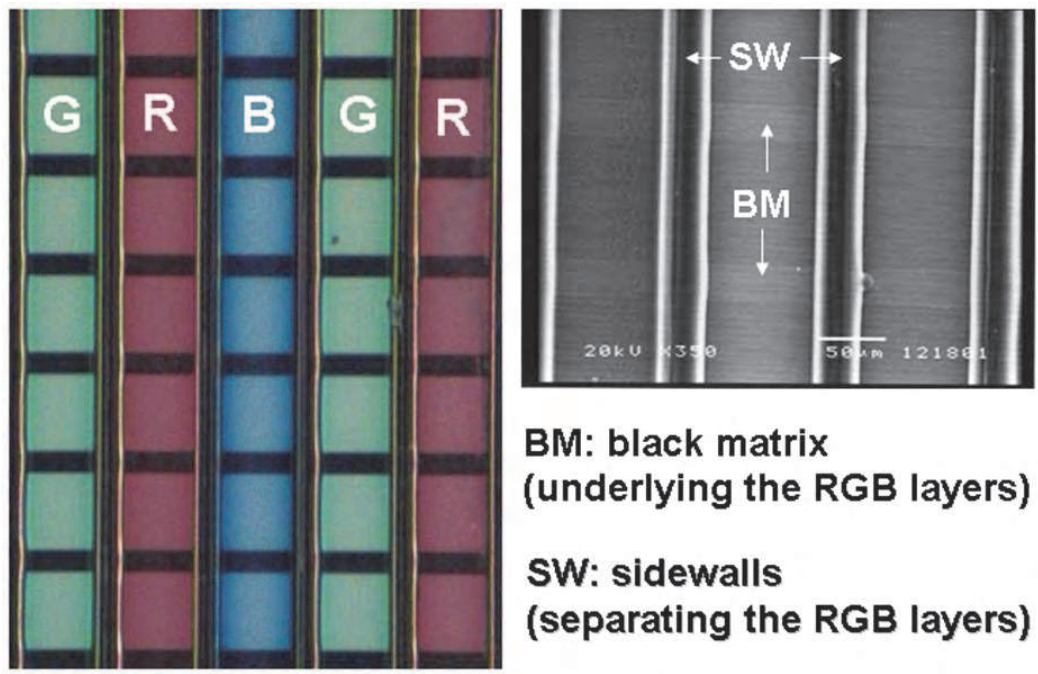

Fig. 9. One inkjet-printed stripe-type LCD color filter with primary colors of red (R), green $(\mathrm{G})$, and blue (B) built on the underlying black-matrix (BM) glass.

being performed. With high flexibility and light weight, the polymer LED display is one of promising candidates for low power consumption in the near future, particularly in the applications of portable consumer devices (e.g., mobile phones and electronic books).

Besides, the LEDs can be enhanced in brightness together with the microlens embedded on top. Figure 10 demonstrates such a lens-cap effect on LEDs, in which the polymer microlenses were deposited to introduce more illumination out of the lighting plane that will be further explained below.
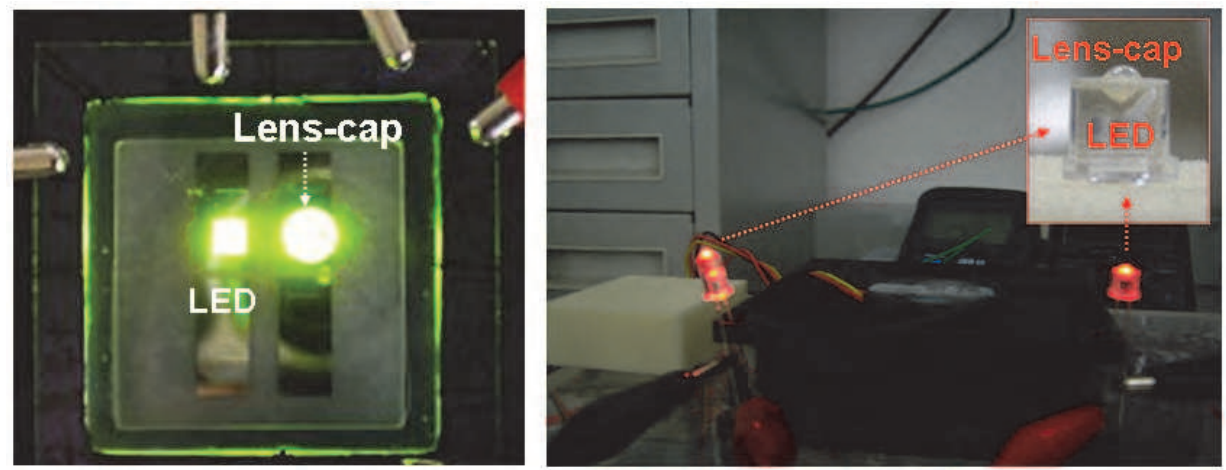

Fig. 10. (Left) Comparison between lens-less and lens-cap green light-emitting diodes (LEDs) showing the lens effect of brightness enhancement; (Right) lens-cap red LEDs.

\subsection{Microlenses and back light planes}

The inkjet-printed microlenses was introduced in 1994 when MacFarlane et al. published their works on microjet fabrication of microlens arrays for collimating light beam 
(MacFarlane et al., 1994). Since then, the refractive microlenses were widely investigated by direct inkjet printing for more functionality with incorporation of other devices such as LEDs and VCSEL (Jeon et al., 2005; Nallani et al., 2006). As shown previously in Figure 8, the microlenses feature three-dimensional (3D) curvatures of hemispherical shapes, significantly different from those thin-film layers for CFs and LEDs. As evaporative inks used herein for polymer lenses, the $\mathrm{CR}$ effect should be treated in inkjet printing by modifying the substrate surface energy (Chen et al., 2008).

In addition, one potential application for microlenses is associated to the back light plane that transports light of source from the back (side) to front surface of plane by virtue of lens curvature. Nevertheless, compared to conventional techniques of fabrication such as molding and injection, this application is limited to hemispherical profile of a lens, and therefore suffers significantly from low coverage of inkjet printing on plane surface that needs to be further improved in the future.

\subsection{Conductive lines and electrodes}

Besides the light emitting or transport in CFs, LEDs, and microlenses, both the conductive lines and electrodes are basic elements in electricity delivery for electronic devices. Mostly, with synthesis of nanoparticle metals instead of polymers for inks, the electrical properties of inkjet-printed conductors have been investigated recently in many researches (Fuller et al., 2002; Lee et al., 2005; Kang et al., 2010; Scandurra et al. 2010). Because of the need for fusing the nanoparticles, those inkjet print of metal inks typically feature a sintering process at elevated temperature $\left(>100{ }^{\circ} \mathrm{C}\right)$ to reduce their porous portions of structure, in which the resistivity of printed materials can be as low as 5-7×10-6 $\Omega \mathrm{cm}$ (Scandurra et al. 2010).

Furthermore, this type of conductive elements can be commonly applied in flexible microelectronics that has been attracting many efforts in recent years (Perelaer \& Schubert, 2010). As demonstrated in Figure 11, the conductive Ag (silver) lines and electrodes can be directly inkjet printed and sintered on a flexible PET (Polyethylene terephthalate) substrate using a commercial Dimatix material printer (DMP 2800). Similarly, electric transistors and integrated circuits can be fulfilled as below.
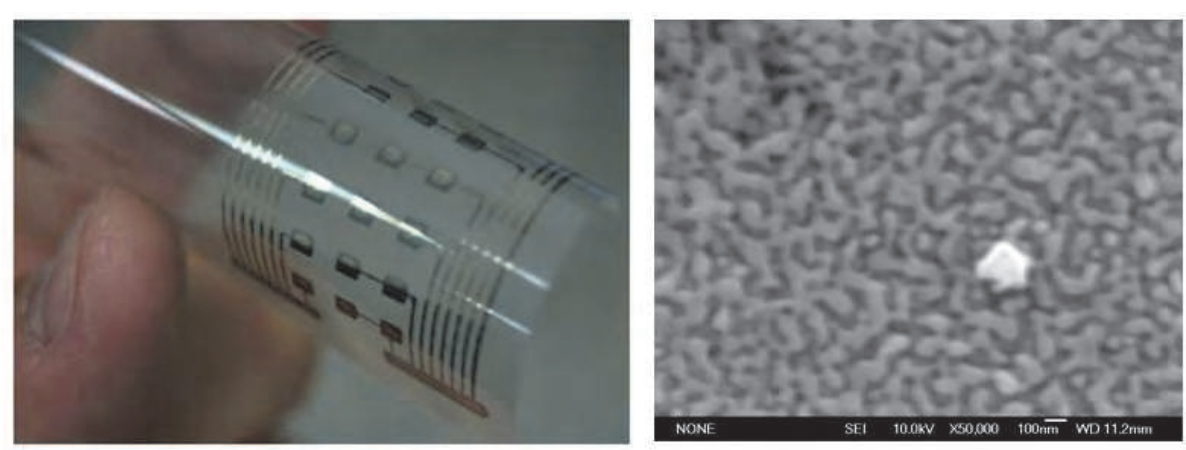

Fig. 11. (Left) Electronic conductors inkjet-printed on a highly flexible PET substrate using Ag-nanoparticle solutions; (Right) the surface morphology of the conductors after sintering at $250^{\circ} \mathrm{C}$. 


\subsection{Transistors and integrated circuits}

Ultimate aim in the field of the inkjet-printed microelectronics is no doubt led to fully fabricate the transistors and integrated circuits that is still at early stage of development in scientific researches (Sirringhaus et al., 2000; Han et al., 2009; Lim et al., 2010; Hinemawari et al., 2011). This revolutionary development, in science and technique as well, can be eventually conducted into the many applications including the thin-film transistor liquid crystal display (TFT-LCD).

Interestingly, more other technical disciplines and ideas, such as soft-lithography and selfassembly (Bruzewicz et al., 2008; Chen et al., 2011), are being gradually blended into inkjet printing of microcomponents, whereby perhaps generating a novel phase for microfabrication in the future (see Figure 12).
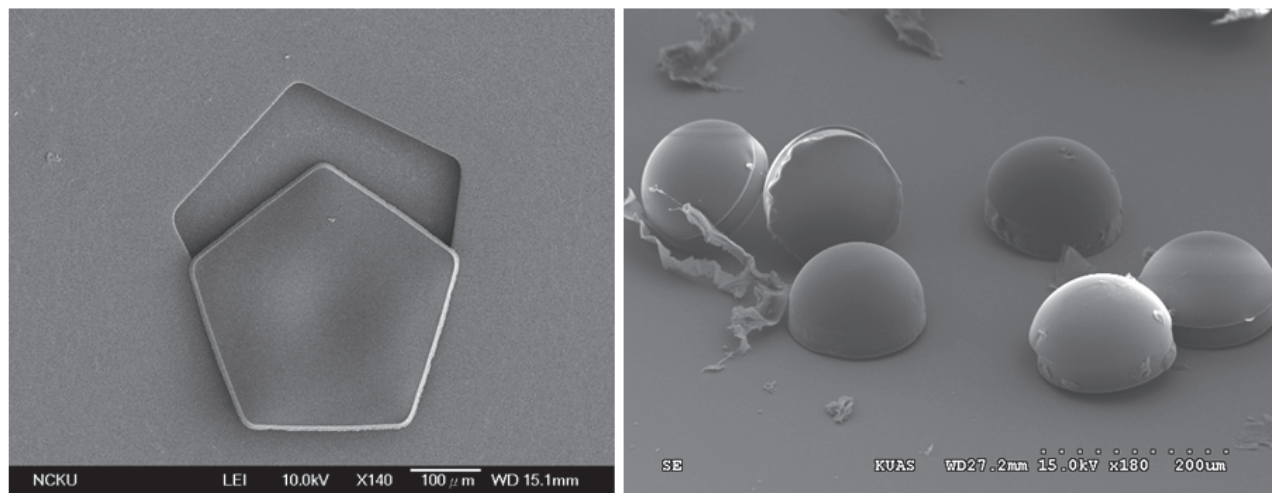

Fig. 12. (Left) one 5-sided regular polygon inkjet-printed and self-formed from a micro cavity; (Right) multiple hemispherical polymer microstructures inkjet-printed and selfleased from their corresponding master molds.

\section{Concluding remarks}

Indeed, the DOD inkjet printing technology has proved, in recent decades, a powerful tool for digital microfabrication. Key success elements for fulfilling quality inkjet printing involve availabilities and selections of ink materials, substrates, droplet generation, platform and algorithm. Technical issues such as positioning accuracy and morphology formation should be well dealt with in good design, which strongly rely on the full understanding of fundamental fluidics and mechanics.

Droplet depositions, including evaporation deposit and pattern, will eventually find most suitable applications, in which LCD color filters, polymer LEDs, microlenses, conductors, transistors and integrated circuits have been demonstrated using the inkjet printing technique. In the future, this developing technique fused with other disciplines may open novel routes to fabricate more versatile microcomponents.

\section{Acknowledgements}

The author thanks research grants for this work partially by the National Science Council (NSC) under NSC-99-2221-E-151-034 and NSC-100-2221-E-151-042, Taiwan, ROC. 


\section{References}

Adachi, E.; Dimitrov, A. S. \& Nagayama, K. (1995). Stripe patterns formed on a glass surface during droplet evaporation. Langmuir, 11, 1057-1060.

Alfeeli, B.; Cho, D.; Ashraf-Khorassani, M.; Taylor, L. T. \& Agah, M. (2008). MEMS-based multi-inlet/outlet preconcentrator coated by inkjet printing of polymer adsorbents. Sens. Actuators B, 133, 24-32.

Bale, M.; Carter, J. C.; Creighton, C. J.; Gregory, H. J.; Lyon, P. H.; Ng, P.; Webb, L. \& Wehrum, A. (2006). Ink-jet printing: the route to production of full-color P-OLED displays. J. Soc. Inf. Display, 15, 453-459.

Bao, Z.; Chen, L.; Weldon, E. C.; Cherniavskaya, O.; Dai, Y. \& Tok, J. B.-H. (2002). Toward controllable self-assembly of microstructures: selective functionalization and fabrication of patterned spheres. Chem. Mater., 14, 24-26.

Biehl, S.; Danzebrink, R.; Oliveira, P. \& Aegerter, M. A. (1998). Refractive microlens fabrication by inkjet-jet process. J. Sol-Gel Sci. Tech., 13, 177-182.

Biswas, S.; Gawande, S.; Bromberg V. \& Sun, Y. (2010). Effects of particle size and substrate surface properties on deposition dynamics of inkjet-printed colloidal drops for printable photovoltaics fabrication. J. Sol. Energy Eng., 132, 021010 (7 pages).

Bourges-Monnier, C. \& Shanaha, M. E. R. (1995). Influence of evaporation on contact angle. Langmuir, 11, 2820-2829.

Bruzewicz, D. A.; Reches, M. \& Whitesides, G. M. (2008). Low-cost printing of poly(dimethylsiloxane) barriers to define microchannels in paper. Anal. Chem., 80, 3387-3392.

Busato, S.; Belloli, A. \& Ermanni, P. (2007). Inkjet printing of palladium catalyst patterns on polyimide film for electroless copper plating. Sens. Actuators B, 123, 840-846.

Chang, C.-J.; Chang, S.-J. ; Wu, F.-M. ; Hsu, M.-W. ; Chiu, W. W. W. \& Chen, K. (2004). Effect of compositions and surface treatment on the jetting stability and color uniformity of ink-jet printed color filter. Jpn. J. Appl. Phys., 43, 8227-8233.

Chang, C.-J.; Lin, Y.-H. \& Tsai, H.-Y. (2011). Synthesis and properties of UV-curable hyperbranched polymers for ink-jet printing of color micropatterns on glass. Thin Solid Films, 519, 5243-5248.

Chen, C.-T. \& Yang, T.-Y. (2001). Flow behavior of micro inkjet drop on the layer surface of micro color device. Proc. of NIP, 17, 776-779.

Chen, C.-T. (2004). Experimental and numerical study of thin-film formation by microfluidic deposition method. Proc. of SPIE, 5519, 255-261.

Chen, C.-T.; Tseng, F.-G.; Chieng, C.-C. (2006). Evaporation evolution of volatile liquid droplets in nanoliter wells. Sens. Actuators A, 130-131, 12-19.

Chen, C.-T.; Hsu, C.-Y. \& Chiu, C.-L. (2007). Striped droplet deposition on patterned surfaces using inkjet-printing method. Tamkang J. of Sci. Eng., 10, 107-112.

Chen, C.-T.; Chiu, C.-L.; Tseng, Z.-F. \& Chuang, C.-T. (2008). Dynamic evolvement and formation of refractive evaporative polyurethane droplets. Sens. Actuators A, 147, 369-377.

Chen, C.-T.; Tseng, Z.-F.; Chiu, C.-L.; Hsu, C.-Y. \& Chung, C.-T. (2009). Self-aligned hemispherical formation of microlenses from colloidal droplets on heterogeneous surfaces. J. Micromech. Microeng., 19, 025002 (9pp).

Chen, C.-T.; Wu, K.-H.; Lu, C.-F. \& Shieh, F. (2010). An inkjet printed stripe-type color filter of liquid crystal display. J. Micromech. Microeng., 20, 005004 (11pp).

Chen, C.-T.; Chiu, C.-L.; Hsu, C.-Y.; Tseng, Z.-F. \& Chuang, C.-T. (2011). Inkjet-printed polymeric microstructures in n-sided regular polygonal cavities. J. Microelectromech. Syst. , 20, 1001-1009. 
Chiu, C.-L. \& Chen, C.-T. (2006). The concept and prototype system of medicine-jet capsule endoscope. Opt. Quantum Electron., 37, 1447-1456.

Cho, H.; Parameswaran, M. \& Yu, H.-Z. (2007). Fabrication of microsensors using unmodified office inkjet printers. Sens. Actuators B, 123, 749-756.

Conway, J.; Korns, H. \& Fisch, M. R. (1997). Evaporation kinematics of polystyrene bead suspensions. Langmuir, 13, 426-431.

Darhuber, A. A.; Troian, S. M.; Miller, S. M. \& Wager, S. (2000). Morphology of liquid microstructures on chemically patterned surfaces. J. Appl. Phys., 87, 7768-7775.

Decker, E. L. \& Garoff, S. (1997). Contact line structure and dynamics on surfaces with contact angle hysteresis. Langmuir, 13, 6321-6332.

Deegan, R. D.; Bakajin, O.; Dupont, T. F.; Huber G.; Nagel, S. R. \& Witten T. A. (1997). Capillary flow as the cause of ring stains from dried liquid drops. Nature, 389, 827-829.

de Gans B.-J.; Duineveld, P. C. \& Schubert, U. S. (2004). Inkjet printing of polymers: state of the art and future developments. Adv. Mater., 16, 203-213.

Erbil, H. Y.; McHale, G. \& Newton, M. I. (2002). Drop evaporation on solid surfaces: constant angle mode. Langmuir, 18, 2636-2641.

Fuchs, G.; Diges, C.; Kohlstaedt, L. A.; Wehner, K. A. \& Sarnow, P. (2011). Proteomic analysis of ribosomes: translational control of mRNA populations by glycogen synthase GYS1. J. Mol. Biol., 410, 118-130.

Fuller, S. B.; Wilhelm, E. J. \& Jacobson, J. M. (2002). Ink-jet printed nanoparticle microelectromechanical systems. J. Microelectromech. Syst., 11, 54-60.

Gau, H.; Herminghaus, S.; Lenz, P. \& Lipowsky, R. (1999). Liquid morphologies on structured surfaces: from microchannels to microchips. Science, 283, 46-49.

Gorand, Y.; Pauchard, L.; Calligari, G.; Hulin, J. P. \& Allain, C. Mechanical instability induced by the desiccation of sessile drops. Langmuir, 20, 5138-5140.

Gutmann, O.; Kuehlewein, R.; Reinbold, S.; Niekrawietz, R.; Steinert, C. P.; de Heij, B.; Zengerle, R. \& Daub, M. (2005). Fast and reliable protein microarray production by a new drop-in-drop technique. Lab Chip, 5, 675-681.

Han, S.-Y.; Lee, D.-H.; Herman, G. S. \& Chang C.-H. (2009). Inkjet-printed high mobility transparent-oxide semiconductors. J. Display Tech., 5, 520-524.

Hinemawari, H.; Yamada, T.; Matsui, H.; Tsutsumi, J.; Haas, S.; Ryosuke, C. \& Hasegawa, T. (2011). Inkjet printing of single-crystal films. Nature, 475, 364-367.

Hu, H. \& Larson, R. G. (2002). Evaporation of a sessile droplet on a substrate. J. Phys. Chem., $106,1334-1344$.

Jeon, C. W.; Gu, E.; Liu, C.; Girkin, J. M. \& Dawson, M. D. (2005). Polymer microlens arrays applicable to AlInGaN ultraviolet micro-light-emitting diodes. IEEE Photo. Technol. Lett., 17, 1887-1889.

Joshi, A. S. \& Sun Y. (2010). Numerical simulation of colloidal drop deposition dynamics on patterned substrates for printable electronics fabrication. J. Display Tech., 6, 579-585.

Kang, J. S.; Kim, H. S.; Ryu, J.; Hahn, H. T.; Jang, S. \& Joung, J. W. (2010). Inkjet printed electronics using copper nanoparticle ink, J. Mater. Sci: Mater. Electron., 21, 1213-1220.

Katayama, M. (1999). TFT-LCD technology. Thin Solid Films, 341, 140-147.

Kim, E. \& Whitesides, G. M. (1995). Use of minimal free energy and self-assembly to form shapes. Chem. Mater., 7, 1257-1264.

Kim, Y. D.; Kim, J. P.; Kwon, O. S. \& Cho, I. H. (2009). The synthesis and application of thermally stable dyes for ink-jet printed LCD color filters. Dyes Pigments, 81, 45-52.

Koo, H. S.; Chen, M.; Pan., P. C.; Chou, L. T.; Wu, F. M.; Chang, S. J. \& Kawai, T. (2006). Fabrication and chromatic characteristics of the greenish LCD colour-filter layer with nano-particle ink using inkjet printing technique. Displays, 27, 124-129. 
Le, H. P. (1998). Progress and trends in ink-jet printing technology. J. Imaging Sci. Technol., 42, 49-62.

Lee, H.-H.; Chou, K.-S. \& Huang, K.-C. (2005). Inkjet printing of nanosized silver colloids. Nanotechnology, 16, 2436-2441.

Lenz, P.; Fenzl, W. \& Lipowsky, R. (2011). Wetting of ring-shaped surface domains. Europhys. Lett., 53, 618-624.

Lim, J. A.; Kim, J.-H.; Qiu, L.; Lee W. H.; Lee, H. S.; Kwak, D. \& Cho, K. (2010). Inkjet-printed single-droplet organic transistors based on semiconductor nanowires embedded in insulating polymers. Adv. Func. Mater., 20, 3292-3297.

Liou, T.-M.; Chan, C.-Y.; Fu, C.-C. \& Shih, K.-C. (2008). Effects of impact inertia and surface characteristics on deposited polymer droplets in microcavities. J. Microelectromech. Syst., 17, 278-287.

MacFarlane, D. L.; Narayan, V.; Tatum, J. A.; Cox, W. R.; Chen, T. \& Hayes, D. J. (1994). Microjet fabrication of microlens arrays. IEEE Photonics Tech. Lett., 6, 1112-1114.

Mentley, D. E. (2002). State of flat-panel display technology and future trends. Proc. of the IEEE, 90, 453-459.

Nallani, A. K.; Chen, T.; Hayes, D. J.; Che, W.-S. \& Lee, J.-B. (2006). A method for improved VCSEL packaging using MEMS and ink-jet technologies. J. Lightw. Technol., 24, 1504-1512.

Parisse, F. \& Allain, C. (1997). Drying of colloidal suspension droplets: experimental study and profile renormalization. Langmuir, 13, 3598-3602.

Perelaer, J. \& Schubert, U. S. (2010). Inkjet printing and alternative sintering of narrow conductive tracks on flexible substrates for plastic electronic applications, INTECH, ISBN 978-9537619-72-5, Book Chapter 16.

Satoi, T. (2001). Color filter manufacturing apparatus. US Patent 6,331,384 B1.

Scandurra, A.; Indelli, G. F.; Sparta, N. G.; Galliano, F.; Ravesi, S. \& Pignatro S. (2010). Lowtemperature sintered conductive silver patterns obtained by inkjet printing for plastic electronics. Surf. Interface Annal., 42, 1163-1167.

Shaw, J. M. \& Seidler, P. F. (2001). Organic electronics: introduction. IBM J. Res. E Dev., 45, 3-9.

Sirringhaus, H.; Kawase, T.; Friend, R. H.; Shimoda, T.; Inbasekaran, M.; Wu. W. \& Woo, E. P. (2000). High-resolution inkjet printing of all-polymer transistor circuits. Science, 290, 2123-2126.

Soltman, D. \& Subramanian, V. (2008). Inkjet-printed line morphologies and temperature control of the coffee ring effect. Langmuir, 24, 2224-2231.

Szczech B. J.; Megaridis, C. M.; Gamota, D. R. \& Zhang, J. (2002). Fine-line conductor manufacturing using drop-on-demand PZT printing technology. IEEE Trans. Electron. Packag. Manufact., 25, 26-33.

Tseng, F.-G.; Kim, C.-J.; Ho, C.-M. (2002). A high-resolution high-frequency monolithic topshooting microinjector free of satellite drops - part I: concept, design, and model. J. Microelectromech. Syst. , 11, 427-436.

van der Vaart, N. C.; Lifka, H.; Budzelaar, F. P. M.; Rubingh, J. E. J. M.; Hoppenbrouwers, J. J. L.; Dijksman, J. F.; Verbeek, R. G. F. A.; van Woudenberg, R.; Vossen, F. J.; Hiddink, M. G. H.; Rosink, J. J. W. M.; Bernards, T. N. M.; Giraldo, A.; Young, N. D.; Fish, D. A.; Childs, M. J.; Steer, W. A. \& George, D. S. (2005). Towards large-area full-color activematrix printed polymer OLED television. J. Soc. Inf. Display, 13/1, 9-16.

Weon, B. M. \& Je, J. H. (2010). Capillary force repels coffee-ring effect. Phys. Rev. E, 82, 015305(R) (4 pages).

$\mathrm{Xu}$, T.; Jin, J.; Gregory, C.; Hickman, J. J. \& Boland, T. (2005). Inkjet printing of viable mammalian cells. Biomaterials, 26, 93-99. 


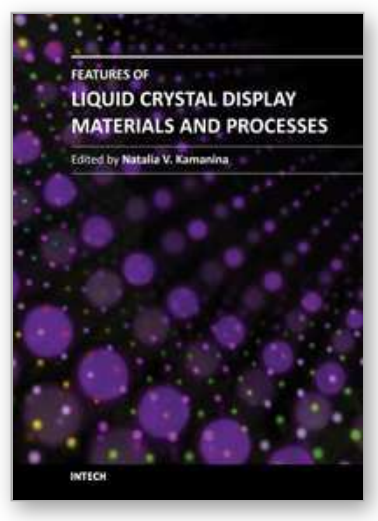

\section{Features of Liquid Crystal Display Materials and Processes}

Edited by Dr. Natalia Kamanina

ISBN 978-953-307-899-1

Hard cover, 210 pages

Publisher InTech

Published online 30, November, 2011

Published in print edition November, 2011

Following the targeted word direction of Opto- and Nanoelectronics, the field of science and technology related to the development of new display technology and organic materials based on liquid crystals ones is meeting the task of replacing volume inorganic electro-optical matrices and devices. An important way in this direction is the study of promising photorefractive materials, conducting coatings, alignment layers, as well as electric schemes that allow the control of liquid crystal mesophase with good advantage. This book includes advanced and revised contributions and covers theoretical modeling for optoelectronics and nonlinear optics, as well as includes experimental methods, new schemes, new approach and explanation which extends the display technology for laser, semiconductor device technology, medicine, biotechnology, etc. The advanced idea, approach, and information described here will be fruitful for the readers to find a sustainable solution in a fundamental study and in the industry.

\section{How to reference}

In order to correctly reference this scholarly work, feel free to copy and paste the following:

Chin-Tai Chen (2011). Inkjet Printing of Microcomponents: Theory, Design, Characteristics and Applications, Features of Liquid Crystal Display Materials and Processes, Dr. Natalia Kamanina (Ed.), ISBN: 978-953-307899-1, InTech, Available from: http://www.intechopen.com/books/features-of-liquid-crystal-display-materialsand-processes/inkjet-printing-of-microcomponents-theory-design-characteristics-and-applications

\section{INTECH}

open science | open minds

\section{InTech Europe}

University Campus STeP Ri

Slavka Krautzeka 83/A

51000 Rijeka, Croatia

Phone: +385 (51) 770447

Fax: +385 (51) 686166

www.intechopen.com

\section{InTech China}

Unit 405, Office Block, Hotel Equatorial Shanghai

No.65, Yan An Road (West), Shanghai, 200040, China

中国上海市延安西路65号上海国际贵都大饭店办公楼 405 单元

Phone: +86-21-62489820

Fax: $+86-21-62489821$ 
(C) 2011 The Author(s). Licensee IntechOpen. This is an open access article distributed under the terms of the Creative Commons Attribution 3.0 License, which permits unrestricted use, distribution, and reproduction in any medium, provided the original work is properly cited. 